PROCEEDINGS OF THE

AMERICAN MATHEMATICAL SOCIETY

Volume 137, Number 9, September 2009, Pages 2913-2919

S 0002-9939(09)09864-5

Article electronically published on March 5, 2009

\title{
LEAST TOTIENTS IN ARITHMETIC PROGRESSIONS
}

\author{
JAVIER CILLERUELO AND MOUBARIZ Z. GARAEV
}

(Communicated by Wen-Ching Winnie Li)

\begin{abstract}
Let $N(a, m)$ be the least integer $n$ (if it exists) such that $\varphi(n) \equiv a$ $(\bmod m)$. Friedlander and Shparlinski proved that for any $\varepsilon>0$ there exists $A=A(\varepsilon)>0$ such that for any positive integer $m$ which has no prime divisors $p<(\log m)^{A}$ and any integer $a$ with $\operatorname{gcd}(a, m)=1$, we have the bound $N(a, m) \ll m^{3+\varepsilon}$. In the present paper we improve this bound to $N(a, m) \ll$ $m^{2+\varepsilon}$.
\end{abstract}

\section{INTRODUCTION}

The distribution properties of the values of Euler's function $\varphi(n)$ in arithmetic progressions have been studied in a series of papers; see for example [1]- 5 . Friedlander and Shparlinski investigated the size of the least integer $n$, to be denoted by $N(a, m)$, such that

$$
\varphi(n) \equiv a \quad(\bmod m)
$$

They proved that if $m=q$ is a prime number, then $N(a, q) \ll q^{5 / 2+\varepsilon}$, which afterwards was improved by Garaev to $N(a, q) \ll q^{2+\varepsilon}$. In the case of a composite number modulo $m$, Friedlander and Shparlinski established that for some $A=$ $A(\varepsilon)>0$ if $(a, m)=1$ and if $m$ has no prime divisors $p<(\log m)^{A(\varepsilon)}$, then $N(a, m) \ll m^{3+\varepsilon}$. The aim of the present paper is to improve this bound further to $N(a, m) \ll m^{2+\varepsilon}$, which at the same time extends Garaev's bound to this class of composites modulo $m$.

Theorem 1.1. For any $\varepsilon>0$ there exists $A=A(\varepsilon)>0$ such that, uniformly for integers $m \geq 1$ which have no prime divisors $p<(\log m)^{A}$ and a with $(a, m)=1$, we have the bound

$$
N(a, m) \ll m^{2+\varepsilon} .
$$

In the opposite direction, the result of Friedlander and Luca [3] implies that there exists a sequence of arithmetical progressions $a_{k}\left(\bmod m_{k}\right)$ with $m_{k} \rightarrow \infty$ as $k \rightarrow \infty$ such that $N\left(a_{k}, m_{k}\right)$ exists and

$$
\frac{\log N\left(a_{k}, m_{k}\right)}{\log m_{k}} \rightarrow \infty \quad \text { as } \quad k \rightarrow \infty .
$$

Received by the editors October 28, 2008, and, in revised form, December 18, 2008, and December 22, 2008.

2000 Mathematics Subject Classification. Primary 11B50, 11L40; Secondary 11N64.

During the preparation of this paper, the first author was supported by Grant MTM 2005-04730 of MYCIT.

(C)2009 American Mathematical Society Reverts to public domain 28 years from publication 


\section{THE PROOF}

As in the paper of Friedlander and Shparlinski, we look for a solution of the congruence in question in the form $n=p_{1} p_{2} p_{3}$, where the $p_{j}$ are prime numbers that run through prime numbers of certain disjoint intervals.

Let $k \geq 2$ be a fixed positive integer constant. Let $I_{1}, I_{2}, I_{3}$ be sets of primes defined as follows:

$$
\begin{aligned}
& I_{1}=\left\{p: 0.5 m^{1+1 / k}<p \leq m^{1+1 / k},(p-1, m)=1\right\} \\
& I_{2}=\{p: 0.5 m<p \leq m,(p-1, m)=1\} \\
& I_{3}=\left\{p: 0.5 m^{1 / k}<p \leq m^{1 / k},(p-1, m)=1\right\} .
\end{aligned}
$$

The sets $I_{1}, I_{2}, I_{3}$ are pairwise disjoint for any sufficiently large integer $m$. We will prove that if $m$ is a large integer with no prime divisors less than $(\log m)^{2(k+3)^{2}}$ and if $(a, m)=1$, then the congruence

$$
\left(p_{1}-1\right)\left(p_{2}-1\right)\left(p_{3}-1\right) \equiv a \quad(\bmod m), \quad p_{j} \in I_{j}, j=1,2,3
$$

has solutions. The number $J$ of solutions of this congruence is equal to

$$
J=\frac{1}{\varphi(m)} \sum_{\chi} \sum_{p_{1}, p_{2}, p_{3}} \chi\left(\left(p_{1}-1\right)\left(p_{2}-1\right)\left(p_{2}-1\right)\right) \bar{\chi}(a),
$$

where $\chi$ runs through all multiplicative characters modulo $m$ and the primes $p_{1}$, $p_{2}, p_{3}$ belong to the sets $I_{1}, I_{2}, I_{3}$, respectively. Thus

$$
J=\frac{\left|I_{1}\right|\left|I_{2}\right|\left|I_{3}\right|}{\varphi(m)}+\frac{\theta}{\varphi(m)} \sum_{\chi \neq \chi_{0}}\left|S_{1}(\chi)\right|\left|S_{2}(\chi)\right|\left|S_{3}(\chi)\right|, \quad|\theta| \leq 1,
$$

where

$$
S_{j}(\chi)=\sum_{p \in I_{j}} \chi(p-1), j=1,2,3 .
$$

To prove that $J>0$ it is enough to prove that $\sum_{\chi \neq \chi_{0}}\left|S_{1}(\chi)\right|\left|S_{2}(\chi)\right|\left|S_{3}(\chi)\right|<$ $\left|I_{1}\right|\left|I_{2}\right|\left|I_{3}\right|$.

\subsection{Preliminary lemmas.}

Lemma 2.1. The following bounds hold:

$$
\left|I_{1}\right| \gg \frac{m^{1 / k} \varphi(m)}{\log m}, \quad\left|I_{2}\right| \gg \frac{\varphi(m)}{\log m}, \quad\left|I_{3}\right| \gg \frac{m^{1 / k}}{\log m} \frac{\varphi(m)}{m} .
$$

Proof. This follows easily from [4, Lemma 4].

Lemma 2.2. The following bounds hold:

$$
\begin{aligned}
\sum_{\chi}\left|S_{j}(\chi)\right|^{2} & \ll(\log m)\left|I_{j}\right|^{2}, j=1,2, \\
\sum_{\chi}\left|S_{3}(\chi)\right|^{2 k} & \ll \phi(m) m(\log m)^{k^{2}-1} .
\end{aligned}
$$

Proof. We easily check that

$$
\sum_{\chi}\left|S_{j}(\chi)\right|^{2}=\varphi(m) J_{j}, \quad j=1,2,
$$

where $J_{j}$ is the number of pairs $\left(p, p^{\prime}\right), p, p^{\prime} \in I_{j}$ such that $p \equiv p^{\prime}(\bmod m)$. 
In the case of $j=2$, since $\left|p-p^{\prime}\right|<m$ implies that $p^{\prime}=p$ for those pairs, so the number of pairs is exactly $\left|I_{2}\right|$. Lemma 2.1 gives

$$
\sum_{\chi}\left|S_{2}(\chi)\right|^{2}=\varphi(m) J_{2}=\varphi(m)\left|I_{2}\right|=\frac{\varphi(m)}{\left|I_{2}\right|}\left|I_{2}\right|^{2} \ll(\log m)\left|I_{2}\right|^{2} .
$$

In the case of $j=1$, since $\left|p-p^{\prime}\right|<m^{1+1 / k}$, for each $p$, the number of primes $p^{\prime}$ with $p^{\prime} \equiv p(\bmod m)$ is at most $m^{1 / k}$. Thus $J_{1} \ll m^{1 / k}\left|I_{1}\right|$ and again by Lemma 2.1.

$$
\sum_{\chi}\left|S_{1}(\chi)\right|^{2} \ll \varphi(m) m^{1 / k}\left|I_{1}\right| \leq \frac{\varphi(m) m^{1 / k}}{\left|I_{1}\right|}\left|I_{1}\right|^{2} \ll(\log m)\left|I_{1}\right|^{2} .
$$

To prove (2.3) we observe that

$$
\sum_{\chi}\left|S_{3}(\chi)\right|^{2 k}=\varphi(m) J_{3},
$$

where $J_{3}$ is the number of $\left(p_{1}, \ldots, p_{k}, p_{1}^{\prime}, \ldots, p_{k}^{\prime}\right)$ with $p_{i}, p_{i}^{\prime} \in I_{3}$ such that

$$
\left(p_{1}-1\right) \cdots\left(p_{k}-1\right) \equiv\left(p_{1}^{\prime}-1\right) \cdots\left(p_{k}^{\prime}-1\right) \quad(\bmod m) .
$$

Since both products are less than $m$, the number of solutions of this congruence is bounded by

$$
J_{3} \leq \sum_{n \leq m} \tau_{k}^{2}(m)
$$

where

$$
\tau_{k}(n)=\#\left\{\left(n_{1}, \ldots, n_{k}\right): n_{1} \cdots n_{k}=n\right\}
$$

is the generalized divisor function. Now combining the well-known inequality

$$
\sum_{n \leq m} \tau_{k}^{2}(n) \ll m(\log m)^{k^{2}-1}
$$

with inequalities (2.4) and (2.5), we obtain (2.3).

Lemma 2.3. If $\chi \neq \chi_{0}$, then

$$
\left|S_{1}(\chi)\right| \ll(\log m)^{-k^{2}-6 k-3}(\log \log m)\left|I_{1}\right| .
$$

Proof. We can write

$$
S_{1}(\chi)=\sum_{p \in I_{1}} \chi(p-1)=\sum_{0.5 m^{1+1 / k}<p \leq m^{1+1 / k}} \chi(p-1),
$$

since $\chi(p-1)=0$ when $(p-1, m)>1$. Then

$$
\begin{aligned}
\left|S_{1}(\chi)\right| & =\left|\sum_{p \leq m^{1+1 / k}} \chi(p-1)-\sum_{p \leq 0.5 m^{1+1 / k}} \chi(p-1)\right| \\
& \leq\left|\sum_{p \leq m^{1+1 / k}} \chi(p-1)\right|+\left|\sum_{p \leq 0.5 m^{1+1 / k}} \chi(p-1)\right| .
\end{aligned}
$$

From Rakhmonov's work [6] it is known that if $\chi \neq \chi_{0}$ is a multiplicative character modulo $m$ and $(l, m)=1$, then

$$
\left|\sum_{p \leq x} \chi(p-l)\right| \leq x(\log x)^{5} \tau(q)\left(\sqrt{1 / q+q \tau^{2}\left(q_{1}\right) / x}+x^{-1 / 6} \tau\left(q_{1}\right)\right),
$$


where $q$ is taken modulo the conductor of $\chi, q_{1}=\prod_{p \mid m, p \nmid q} p$ and $\tau$ is the divisor function.

For $x=m^{1+1 / k}$ or $x=0.5 m^{1+1 / k}$, this gives

$$
\begin{aligned}
\left|\sum_{p \leq x} \chi(p-l)\right| & \ll m^{1+1 / k}(\log m)^{5} \frac{\tau(q)}{\sqrt{q}} \\
& +m^{1 / 2+1 /(2 k)}(\log m)^{5} q^{1 / 2} \tau\left(q_{1}\right) \tau(q) \\
& +m^{(1+1 / k) 5 / 6}(\log m)^{5} \tau\left(q_{1}\right) \tau(q) .
\end{aligned}
$$

Since $q \leq m, k \geq 2$ and $\tau\left(q_{1}\right) \tau(q) \leq \tau(m) \ll m^{1 /(4 k)}$ we obtain

$$
\left|\sum_{p \leq x} \chi(p-l)\right| \ll m^{1+1 / k}(\log m)^{5} \frac{\tau(q)}{\sqrt{q}}+m^{1+3 /(4 k)}(\log m)^{5} .
$$

The maximum value of $\frac{\tau(q)}{\sqrt{q}}$ holds when $q$ is the least prime divisor of $m$ that is greater than $(\log m)^{2(k+3)^{2}}$. Thus

$$
\begin{aligned}
\left|\sum_{p \leq x} \chi(p-l)\right| & \ll m^{1+1 / k}(\log m)^{5-(k+3)^{2}}+m^{1+3 /(4 k)}(\log m)^{5} \\
& \ll \frac{m}{\varphi(m)}(\log m)^{6-(k+3)^{2}}\left|I_{1}\right| .
\end{aligned}
$$

Finally we use the known estimate, $\frac{m}{\varphi(m)} \ll \log \log m$.

2.2. End of the proof. Following the idea of [5], we split the set of nonprincipal characters into two subsets:

$$
\begin{aligned}
& \mathcal{A}=\left\{\chi \neq \chi_{0}:\left|S_{3}(\chi)\right| \leq\left|I_{3}\right|(\log m)^{-2}\right\} \\
& \mathcal{B}=\left\{\chi \neq \chi_{0}:\left|S_{3}(\chi)\right|>\left|I_{3}\right|(\log m)^{-2}\right\} .
\end{aligned}
$$

Thus, from (2.1) we have

$$
J=\frac{\left|I_{1}\right|\left|I_{2}\right|\left|I_{3}\right|}{\varphi(m)}+\frac{\theta}{\varphi(m)} \sum_{\mathcal{A}}+\frac{\theta}{\varphi(m)} \sum_{\mathcal{B}}, \quad|\theta| \leq 1,
$$

where

$$
\begin{aligned}
& \sum_{\mathcal{A}}=\sum_{\chi \in \mathcal{A}}\left|S_{1}(\chi)\right|\left|S_{2}(\chi)\right|\left|S_{3}(\chi)\right|, \\
& \sum_{\mathcal{B}}=\sum_{\chi \in \mathcal{B}}\left|S_{1}(\chi)\right|\left|S_{2}(\chi)\right|\left|S_{3}(\chi)\right| .
\end{aligned}
$$

To estimate $\sum_{\mathcal{A}}$ we observe that

$$
\sum_{\mathcal{A}} \leq\left|I_{3}\right|(\log m)^{-2}\left(\sum_{\chi}\left|S_{1}(\chi)\right|^{2}\right)^{1 / 2}\left(\sum_{\chi}\left|S_{2}(\chi)\right|^{2}\right)^{1 / 2} .
$$

Using Lemma 2.2 we get that

$$
\sum_{\mathcal{A}} \ll(\log m)^{-1}\left|I_{1}\right|\left|I_{2}\right|\left|I_{3}\right|
$$


To estimate $\sum_{\mathcal{B}}$, we first note that

$$
\sum_{\mathcal{B}} \leq|\mathcal{B}|\left(\max _{\chi \neq \chi_{0}}\left|S_{1}(\chi)\right|\right)\left|I_{2}\right|\left|I_{3}\right| .
$$

Next we estimate $|\mathcal{B}|$ using Lemma 2.2

$$
|\mathcal{B}|\left|I_{3}\right|^{2 k}(\log m)^{-4 k} \leq \sum_{\chi}\left|S_{3}\right|^{2 k} \ll \varphi(m) m(\log m)^{k^{2}-1} .
$$

Thus

$$
|\mathcal{B}| \ll(\log m)^{k^{2}+4 k-1} \varphi(m) m\left(\frac{m^{1 / k}}{\log m} \frac{\varphi(m)}{m}\right)^{-2 k} \ll(\log m)^{k^{2}+6 k-1}\left(\frac{m}{\varphi(m)}\right)^{2 k-1} .
$$

We use again that $\frac{m}{\varphi(m)} \ll \log \log m$ and Lemma 2.3 to obtain

$$
\begin{aligned}
\sum_{\mathcal{B}} & \ll|\mathcal{B}|(\log m)^{-k^{2}-6 k-3}(\log \log m)\left|I_{1}\right|\left|I_{2}\right|\left|I_{3}\right| \\
& \ll(\log m)^{-4}(\log \log m)^{2 k}\left|I_{1}\right|\left|I_{2}\right|\left|I_{3}\right| .
\end{aligned}
$$

Inserting this estimate together with (2.7) into (2.6), we get that

$$
J=\frac{\left|I_{1}\right|\left|I_{2}\right|\left|I_{3}\right|}{\varphi(m)}\left(1+O\left((\log m)^{-1}\right)\right) \text {. }
$$

Thus, we have proved that for $m$ large enough the congruence

$$
\left(p_{1}-1\right)\left(p_{2}-1\right)\left(p_{3}-1\right) \equiv a \quad(\bmod m)
$$

has some solution $p_{1} \in I_{1}, p_{2} \in I_{2}, p_{3} \in I_{3}$. Since $\left(p_{1}-1\right)\left(p_{2}-1\right)\left(p_{3}-1\right) \leq m^{2+2 / k}$, we finish the proof of our theorem.

\section{Exceptional RESIDUES}

In this section we take into account the referee's suggestion and show that under the conditions of Theorem 1.1 the bound $N(a, m) \ll m^{1+\varepsilon}$ holds for almost all reduced residue classes $a$ modulo $m$.

Theorem 3.1. For any $\varepsilon>0$ there exists $A=A(\varepsilon)>0$ such that, uniformly for integers $m \geq 1$ which have no prime divisors $p<(\log m)^{A}$, the bound

$$
N(a, m) \ll m^{1+\varepsilon}
$$

holds for all but $o(\phi(m))$ reduced residue classes a $(\bmod m)$.

Proof. We use the notation introduced in the proof of Theorem 1.1. Let $H$ be the set of all elements $h \in \mathbb{Z}_{m}^{*}$ such that the congruence

$$
\left(p_{1}-1\right)\left(p_{3}-1\right) \equiv h \quad(\bmod m)
$$

has no solutions in primes $p_{1} \in I_{1}, p_{3} \in I_{3}$. If $|H| \leq\left|I_{2}\right|$, then we are done in view of $\left|I_{2}\right| \ll \frac{m}{\log m}$. If $|H|>\left|I_{2}\right|$, then let $H_{1}$ be a subset of $H$ with $\left|H_{1}\right|=\left|I_{2}\right|$. The number of solutions of the congruence

$$
\left(p_{1}-1\right)\left(p_{3}-1\right) \equiv h \quad(\bmod m), \quad p_{j} \in I_{j}, j=1,3, h \in H_{1}
$$

can be written in the form

$$
J=\frac{\left|I_{1}\right|\left|I_{2}\right|\left|I_{3}\right|}{\varphi(m)}+\frac{\theta}{\varphi(m)} \sum_{\chi \neq \chi_{0}}\left|S_{1}(\chi)\right|\left|S_{2}(\bar{\chi})\right|\left|S_{3}(\chi)\right|, \quad|\theta| \leq 1,
$$


where

$$
S_{j}(\chi)=\sum_{p \in I_{j}} \chi(p-1), j=1,3, \quad S_{2}(\chi)=\sum_{h \in H_{1}} \chi(h) .
$$

Now replacing in the proof of Theorem 1.1 the set $I_{2}$ by $H_{1}$, we obtain

$$
J=\frac{\left|I_{1}\right|\left|I_{2}\right|\left|I_{3}\right|}{\varphi(m)}\left(1+O\left((\log m)^{-1}\right)\right) .
$$

Thus $J \neq 0$, and we get a contradiction.

\section{REMARKS}

We remark that the proof of Theorem 1.1 allows us to eliminate the influence of characters that belong to the set $\mathcal{B}$, while for characters from the set $\mathcal{A}$ we have estimates over very short intervals. This shows that a possible improvement on the length for the interval in Rakhmonov's character sum estimate would not reduce the exponent 2 in the upper bound $N(a, m) \ll m^{2+\varepsilon}$ unless some additional ingredient were introduced. Indeed, in order for the argument to proceed, two dense sets are needed (like $I_{1}$ and $I_{2}$ ) which already contribute the quantity $m^{2+o(1)}$ to the upper bound for $N(a, m)$.

Finally we remark that Theorems 1.1, 3.1 can be put into the following more general statement.

Theorem 4.1. For any $\varepsilon>0$ and for any fixed $r>0$ there exists a number $A=A(\varepsilon, r)>0$ such that, uniformly for integers $m \geq 2$ which have no prime divisors $p<(\log m)^{A}$, the bound

$$
N(a, m) \ll m^{1+\varepsilon}
$$

holds for all but $O\left(m /(\log m)^{r}\right)$ reduced residue classes a $(\bmod m)$.

The proof of Theorem 4.1 follows the same lines as the proofs of Theorems 1.1 and 3.1 and we leave it to the interested reader as an exercise.

\section{ACKNOWLEDGMENT}

The authors wish to thank the referee for useful remarks and suggestions.

\section{REFERENCES}

1. T. Dence and C. Pomerance, Euler's function in residue classes, The Ramanujan J. 2 (1998) 7-20. MR.1642868 (99k:11148)

2. K. Ford, S. Konyagin and C. Pomerance, Residue classes free of values of Euler's function, Number Theory in Progress, vol. 2, K. Gyory, H. Iwaniec, and J. Urbanowicz, eds., de Gruyter, Berlin and New York, 1999, 805-812. MR.1689545 (2000f:11120)

3. J. Friedlander and F. Luca, Residue Classes Having Tardy Totients, Bull. London Math. Soc. (to appear).

4. J. Friedlander and I. Shparlinski, Least totient in a residue class, Bull. London Math. Soc. 39 (2007) 425-432. Corrigendum: Least totient in a residue class, Bull. London Math. Soc. 40 (2008) 532. MR2331570 (2008g:11164) MR2418809 
5. M. Z. Garaev, A note on the least totient of a residue class, The Quarterly Journal of Mathematics, doi:10.1093/qmath/han005.

6. Z. Kh. Rakhmonov, On the distribution of values of Dirichlet characters and their applications, Proc. Steklov Inst. Math. 207 (1995) 263-272. MR1401821 (97f:11068)

Instituto de Ciencias Matemáticas (CSIC-UAM-UC3M-UCM) and Departamento de Matemáticas, Universidad Autónoma de Madrid, Madrid-28049, Spain

E-mail address: franciscojavier.cilleruelo@uam.es

Instituto de Matemáticas, Universidad Nacional Autónoma de México, Campus Morelia, Apartado Postal 61-3 (Xangari), C.P. 58089, Morelia, Michoacán, México

E-mail address: garaev@matmor.unam.mx 